\title{
KJIM
}

\section{Massive ventilator-associated pleural empyema}

\author{
Philipp Jud ${ }^{1}$, Nicole Fink-Neuboeck ${ }^{2}$, and Joerg Lindenmann ${ }^{2}$
}

${ }^{1}$ Division of Angiology, Department of Internal Medicine, ${ }^{2}$ Division of Thoracic Surgery, Department of Surgery, Medical University of Graz, Graz, Austria

Received: October 26, 2017

Revised : November 6, 2017

Accepted: November 6, 2017

\section{Correspondence to}

Philipp Jud, M.D.

Tel: +43-316-385-30174

Fax: +43-316-385-3788

E-mail:philipp.jud@medunigraz.at
A tetraplegic 59-year-old man with invasive home mechanical ventilation administered via tracheostomy was admitted to the intensive care unit due to general discomfort, dyspnea, and low oxygen saturation despite high-flow oxygen therapy. In the chest X-ray, considerable left sided pleural effusion with mediastinal shift towards the right side was detected (Fig. 1). Laboratory findings revealed leukocytosis $\left(15.53 \times 10^{9} / \mathrm{L}\right)$ and elevated C-reactive protein $(131 \mathrm{mg} / \mathrm{L})$. Computed tomography of the chest confirmed a massive left-sided pleural effusion with pleural enhancement of the contrast agent and subsequent atelectasis of the entire lung parenchyma. Additionally, split pleura sign could be detected corresponding to pleural empyema (Fig. 2, white arrows). Moreover, Escherichia coli and Streptococcus intermedius could be detected from bronchoalveolar lavage fluid. Surgical irrigation and meticu-

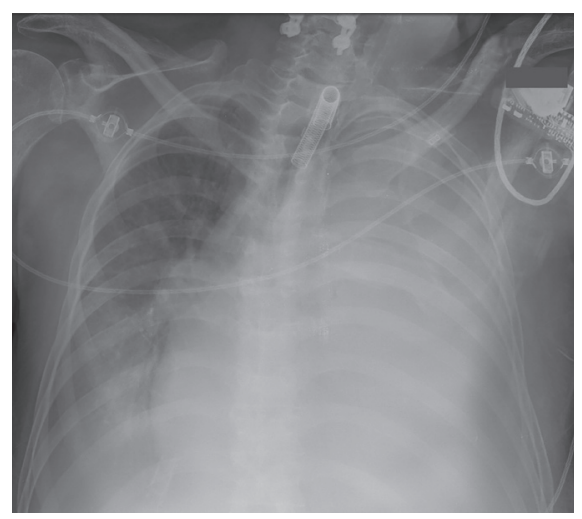

Figure 1. Chest X-ray showing a considerable left sided pleural effusion with subsequent mediastinal shift. lous decortication of the pleural cavity through a left sided posterolateral thoracotomy was performed. Intraoperatively, pleural empyema presented as a green-yellow colored fetid and sticky fluid coating the entire lung surface. Several layers of pus and fibrin were grossly adherent to the parenchyma, mediastinum, and diaphragm. The postoperative course was uneventful, the patient recovered without any sequelae.

Patients with chronic respiratory insufficiency may profit from invasive home mechanical ventilation. However, the airways represent a potential entry for several pathogens enabling severe pulmonary complications like lung abscess or pleural empyema. Split pleura sign is a reliable sign to distinguish empyema from lung abscess in computed tomography, which results

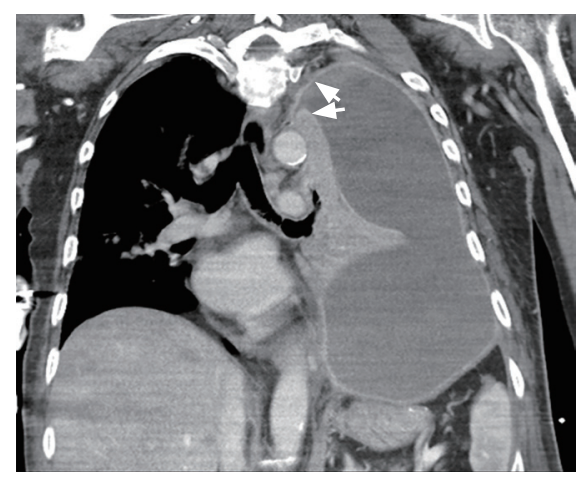

Figure 2. Computed tomography of the chest (frontal plane) confirming massive pleural effusion causing total atelectasis of the left lung. White arrows point toward fibrin-coated parietal and visceral pleura causing 'split pleura sign' corresponding to pleural empyema. 
from fibrin coating of the visceral and parietal pleura with concomitant pleural enhancement. Informed consent was waived by the board.

\section{Conflict of interest}

No potential conflict of interest relevant to this article was reported. 